\title{
Weight Changes According to Treatment in a Diverse Cohort of Breast Cancer Patients
}

\author{
Jami Fukui ( $\nabla$ jfukui@cc.hawaii.edu ) \\ University of Hawaii Cancer Center https://orcid.org/0000-0002-0248-266X \\ Kami White \\ University of Hawai'i Cancer Center \\ Timothy Frankland \\ Kaiser Permanente \\ Caryn Oshiro \\ Kaiser Permanente \\ Lynne Wilkens \\ University of Hawai'i Cancer Center
}

\section{Research article}

Keywords: breast cancer, weight changes, sarcopenia, geriatric oncology, race/ethnicity

Posted Date: July 28th, 2021

DOI: https://doi.org/10.21203/rs.3.rs-260561/v2

License: (c) (1) This work is licensed under a Creative Commons Attribution 4.0 International License. Read Full License 


\section{Abstract}

\section{Background}

Weight changes are common among breast cancer patients. The majority of studies to date have focused on weight gain after a breast cancer diagnosis and its implications on health in survivors. Fewer studies have examined weight loss and its related characteristics. Weight changes have been reported to be influenced by several factors such as age, treatment, stage and pre-diagnostic weight. We evaluated weight changes during key treatment time points in early stage breast cancer patients.

\section{Methods}

We characterized 389 female patients diagnosed in Hawaii with early stage breast cancer from 20032017 in the Multiethnic Cohort (MEC) linked with Kaiser Permanente Hawaii electronic medical record data. We evaluated weight changes from surgery to 4 years post-diagnosis with six time points along a patient's treatment trajectory (chemotherapy, radiation, endocrine, or surgery alone) and annually thereafter, adjusting for age, race/ethnicity and initial body mass index (BMI).

\section{Results}

We found key time points of significant weight change for breast cancer patients according to their adjuvant treatment. In patients who had surgery alone (S), surgery-radiation (SR), or surgery-endocrine therapy (SE), the majority of patients had stable weight, although this consistently decreased over time. However, the percentages of patients with weight loss and weight gain during this time steadily increased up to 4 years after initial surgery. Weight loss was more common than weight gain by about 2 fold in these treatment groups. For patients with surgery-chemotherapy (SC), there was significant weight loss seen within the first 3 months after surgery, during the time when patients receive chemotherapy. And this weight loss persisted until year 4 . Weight gain was less commonly seen in this treatment group.

\section{Conclusions}

We identified key time points during breast cancer treatment that may provide a therapeutic window to positively influence outcomes. Tailored weight management interventions should be utilized to promote overall health and long term survivorship.

\section{Background}

Obesity at diagnosis of breast cancer is an established negative prognostic factor and several studies report an inverse relationship between weight gain post diagnosis and disease free survival (1-3). Weight gain is more common in younger women $(4,5)$ and women receiving adjuvant therapy $(6,7)$.

Schvartsman et al. found age differences related to weight changes during adjuvant chemotherapy, where women over 50 years old were more likely to lose weight during adjuvant chemotherapy, whereas 
women under 30 years old gained weight (8). In one study, Asian women had the lowest risk of weight gain post breast cancer diagnosis (5).

The evaluation of body weight and breast cancer recurrence has been predominantly based on risk associated with excess body weight rather than on the risk associated with weight loss. Sarcopenia, a loss of muscle mass or strength, can naturally occur with aging and is further exacerbated in older adults with cancer. The prevalence of sarcopenia is between $12-57 \%$ in older cancer patients (9). Cancer treatments such as surgery, chemotherapy, and radiation may further contribute to muscle loss (10). Sarcopenia is as common and important a risk factor as obesity for cancer outcomes, including survival, chemotherapy toxicity and surgical outcomes (11). In a recent meta-analysis, sarcopenia was found to be a risk factor for mortality among early stage female breast cancer patients (12). However a study by Del Fabbro et al. examined sarcopenia in women with early stage breast cancer and found it to be associated with improved outcomes (13).

The majority of prior studies evaluated weight change for women at a median age over 55 in early stage breast cancer 6 months or more post diagnosis. Vagenas et. al. found that weight gain can continue to increase with time post-diagnosis, where the percentage overweight or obese was more than half (57\%) of patients at 6 months post-surgery, and increased to $68 \%$ by 72 months post-surgery (6). In a sample of 625 women with early breast cancer, $31 \%$ lost about $5 \mathrm{lbs}, 34 \%$ had a stable weight, and $35 \%$ gained about $5 \mathrm{lbs}$ at two years post-primary treatment (surgical, radiation or chemotherapy) (4). In women diagnosed with stage I - III invasive breast cancer with chemotherapy and/or radiation treatment, $19 \%$ experienced weight loss, $62 \%$ remained stable, and $19 \%$ gained weight at 18 months post diagnosis (14). In a study of Asian women with stage 0 - IV breast cancer, $27 \%$ lost weight, $12 \%$ remained stable, and $61 \%$ gained weight from diagnosis to 18 months post diagnosis (15).

Inconsistency exists between studies that examine weight changes after a breast cancer diagnosis due to variability in population, cancer stage, cancer related treatments, definition of weight change and the time point at which weight change is assessed, making comparisons difficult. Moreover, very few studies examining weight loss after diagnosis and treatment are described in older age groups, over multiple time points, and/or in a multiethnic population, including Native Hawaiians/Pacific Islanders, an understudied group and at high risk for breast cancer incidence and mortality (16).

Our study evaluates changes in weight from surgery to 4 years post-diagnosis, across six time points along a patient's treatment trajectory; chemotherapy, radiation, endocrine treatment, or surgery alone, adjusting for age, race/ethnicity, and BMI at surgery.

\section{Methods}

\section{Study Population}

The Multiethnic Cohort (MEC) was designed to study lifestyle and genetic factors in relation to cancer and other chronic diseases (17). Between 1993-1996 more than 215,000 men and women aged 45-75 
years, mainly African American, Native Hawaiian, Japanese American, Latino, or White, and living in Hawaii or California were enrolled in the MEC by completing a 26-page mailed questionnaire on diet, medical history, and lifestyle. Cancer cases are identified through annual linkage to the statewide SEER registries, the Hawaii Tumor Registry (HTR) and the California Cancer Registry. To improve information on treatment and other aspects of cancer care, a linkage was performed for Hawaii (HI) MEC participants and the Kaiser Permanente Hawaii (KPHI) electronic medical records (EMR) for years 2000-2017. The subset of MEC female breast cancer cases in KPHI with EMR anthropometry data and undergoing surgery is the focus of this manuscript.

Among 2,057 MEC female cancer cases linked to KPHI, 887 were diagnosed with invasive breast cancer and had a first encounter/visit date for that diagnosis (with ICD 9/10 codes for invasive breast cancer) occurring from 2000-2017 (see Fig. 1). Patients with first encounter dates before 2003 were excluded (n $=265$ ) as anthropometric data only became available in the KPHI EMR in that year.

\section{Treatment}

Only patients who had surgery due to breast cancer, primarily lumpectomy or mastectomy during the period from 6 months prior to diagnosis encounter date, to 1 year after, were included $(n=480)$. All treatments received within \pm 1 year of surgery were identified in the EMR. The order of treatments (surgery, chemotherapy, radiation, or endocrine therapy) was determined, and only patients who had surgery as their first treatment were selected for analysis $(n=427)$. Surgery date was assigned as the index date. To evaluate the effect of different treatments on weight outcomes we classified treatment as surgery alone $(\mathrm{S})$, surgery followed by radiation (SR) with and without additional endocrine therapy, surgery followed by chemotherapy (SC), and surgery followed by endocrine therapy (SE).

\section{Weight Measures}

Body mass index (BMI) was first calculated at each patient's encounters using the mean height of all encounters (average number of encounters for the 427 patients with surgery as their first treatment $=21$, mean standard deviation of height values $=0.7$ inches), and used to identify implausible weight measures; in particular weights were excluded if the corresponding BMI value was outside the range of $13-60 \mathrm{~kg} / \mathrm{m}^{2}$. Time from the date of surgery (index date) to the date of measured weight was computed and assigned to the following 7 time points and associated time periods: $0=2$ months prior to and up to the index date, $1=0.1$ to 2.9 months after index date, $2=3$ to 5.9 months after, $3=6$ to 11.9 months after, $4=12$ to 23.9 months after, $5=24$ to 35.9 months after, and $6=36$ to 47.9 months after. Weight measures were averaged within each time period for each patient, and patients who were missing a weight measure at the index time point 0 were excluded. The difference in weight from the index time period was calculated for each subsequent time point and categorized as follows: Lost weight (at least $5 \mathrm{lbs}$ ), Stable (lost/gained within $5 \mathrm{lbs}$ ), and Gained weight (5 lbs or more). The final study sample included 389 patients.

\section{Race/Ethnicity}


Based on KPHI EMR data, patients were classified into four race/ethnic groups for this analysis: Native Hawaiian/Pacific Islander (NHPI), Asian, White, or Other/Unknown. Patients who identified as part American Indian/Alaskan Native or Black or who were unknown $(n=10)$ were assigned "Other/Unknown". The remaining patients were assigned, in priority, as NHPI if they identified as any part Native Hawaiian/Pacific Islander, "Asian" if identified as any part Asian, and "White" if identified as White. We did not consider Hispanic origin in the classification given the low prevalence of patients with Hispanic ethnicity in Hawaii.

\section{Other Measures}

Other covariates of interest from the MEC data included sex, education, neighborhood socioeconomic status (nSES) $(18,19)$, smoking status, dietary intake, physical activity, and reproductive history. The KPHI EMR data was supplemented with HTR data for staging, grade, lymph node involvement, and ER/PR status for the breast cancer diagnosis, as the SEER variables are abstracted and defined uniformly.This study was reviewed and approved by the University of Hawaii and Kaiser Permanente Hawaii Institutional Review Boards for human subjects research.

\section{Statistical analysis}

Mixed generalized odds logistic regression was used to evaluate patient weight changes from surgery (outcomes: lost weight, kept stable weight, gained weight) to six time points. A random intercept term for each patient and fixed effects for time ( 6 levels: $<3,4-5,6-11,12-23,24-35,36-47$ months), age at surgery (3 levels: <65, 65-74, 75 + years), race (4 levels: Native Hawaiian/Pacific Islander, Asian, White, Other/unknown), and BMI at surgery (4 levels: $<25,25-29.9,30-34.5,35+\mathrm{kg} / \mathrm{m}^{2}$ ), were included in the model. The predicted probability for each outcome for each patient was obtained and averaged across all patients for each time period from surgery. To identify possible differences in weight changes by treatment, a similar model was run, including fixed effects for treatment type (4 levels: surgery alone, surgery-chemotherapy, surgery-radiation, surgery-endocrine therapy) and cross-product terms for the interaction of time and treatment type. Fixed effects were assessed using the $F$ test, and two-sided $p$ values $<0.10$ for the interaction term was considered suggestive. All analyses were repeated including only patients with complete data at all time points (overall: $n=248$ patients; by treatment type: surgery alone, $n=33$, surgery-chemotherapy, $n=43$, surgery-radiation, $n=94$, surgery-endocrine therapy, $n=78$ ). Because results from both samples were similar, results from the full sample are reported here. All analyses were performed using SAS version 9.4.

\section{Results}

\section{Sample Characteristics}

As shown in Table 1, the female breast cancer patients were evenly distributed ( 30\%) among Native Hawaiians/Pacific Islanders (NHPI), Asians, and Whites, which is roughly consistent with the racial/ethnic diversity of Hawaii. Mean age at surgery of the overall cohort was 71.1 years with a range of 54-94 
years. NHPI and White patients were younger, whereas Asian patients were older. Mean body mass index (BMI) at surgery was $28.3 \mathrm{~kg} / \mathrm{m}^{2}$ for the entire cohort; NHPI patients had the highest mean BMI and Asian patients the lowest (31.7 and $26.2 \mathrm{~kg} / \mathrm{m}^{2}$, respectively).

Table 1

Patient Characteristics at Breast Cancer Surgery

\begin{tabular}{|c|c|c|c|c|c|}
\hline $\begin{array}{l}\text { Patient Characteristics at } \\
\text { Surgery, } \mathrm{n}(\%)\end{array}$ & All & NHPI & Asian & White & Other/Unknown \\
\hline$N$ & 389 & 118 & 132 & 129 & 10 \\
\hline Age, years, mean (SD) & $\begin{array}{l}71.1 \\
(7.8)\end{array}$ & $\begin{array}{l}69.9 \\
(7.9)\end{array}$ & $\begin{array}{l}73.2 \\
(7.7)\end{array}$ & $\begin{array}{l}70.1 \\
(8.8)\end{array}$ & $71.7(8.8)$ \\
\hline$<65$ & $86(22.1)$ & $31(26.3)$ & $22(16.7)$ & $31(24.0)$ & $2(20.0)$ \\
\hline $65-74$ & $\begin{array}{l}177 \\
(45.5)\end{array}$ & $55(46.6)$ & $53(40.2)$ & $66(51.2)$ & $3(30.0)$ \\
\hline $75+$ & $\begin{array}{l}126 \\
(32.4)\end{array}$ & $32(27.1)$ & $57(43.1)$ & $32(24.8)$ & $5(50.0)$ \\
\hline Height, inches, mean (SD) & $\begin{array}{l}62.6 \\
(2.8)\end{array}$ & $\begin{array}{l}63.6 \\
(2.6)\end{array}$ & $\begin{array}{l}60.6 \\
(2.3)\end{array}$ & $\begin{array}{l}63.8 \\
(2.3)\end{array}$ & $63.1(4.2)$ \\
\hline Weight, lbs, mean (SD) & $\begin{array}{l}158.4 \\
(41.9)\end{array}$ & $\begin{array}{l}182.5 \\
(45.3)\end{array}$ & $\begin{array}{l}136.7 \\
(30.1)\end{array}$ & $\begin{array}{l}157.8 \\
(35.1)\end{array}$ & $167.0(35.1)$ \\
\hline $\mathrm{BMI}, \mathrm{kg} / \mathrm{m}^{2}$, mean (SD) & $\begin{array}{l}28.3 \\
(6.7)\end{array}$ & $\begin{array}{l}31.7 \\
(7.1)\end{array}$ & $\begin{array}{l}26.2 \\
(5.3)\end{array}$ & $\begin{array}{l}27.3 \\
(6.1)\end{array}$ & $29.6(10.5)$ \\
\hline$<25$ & $\begin{array}{l}129 \\
(33.2)\end{array}$ & $18(15.3)$ & $61(46.2)$ & $47(36.4)$ & $3(30.0)$ \\
\hline $25-29.9$ & $\begin{array}{l}126 \\
(32.3)\end{array}$ & $35(29.7)$ & $45(34.1)$ & $43(33.3)$ & $3(30.0)$ \\
\hline $30-34.9$ & $75(19.3)$ & $28(23.6)$ & $18(13.6)$ & $26(20.2)$ & $3(30.0)$ \\
\hline $35+$ & $59(15.2)$ & $37(31.4)$ & $8(6.1)$ & $13(10.1)$ & $1(10.0)$ \\
\hline
\end{tabular}

Table 2 presents characteristics of the patients' breast cancer tumors. All 389 patients had early stage breast cancer, defined as invasive breast cancer in KPHI EMR. The average tumor size was $18.7 \mathrm{~mm}$, with moderately differentiated grade and lymph node negative disease. The majority of patients had estrogen receptor (ER) and progesterone receptor (PR) positive disease (84.1\% and $74.0 \%$, respectively) with $40.4 \%$ of cases having unknown HER2 status. NHPI patients were more likely to have regional stage, when compared to Asian and White patients ( $28 \%$ vs $20 \%$ and $19 \%$, respectively), and to have lymph node positive disease ( $26 \%$ vs $17 \%$ and $16 \%$, respectively). 
Table 2

Tumor Characteristics

\begin{tabular}{|c|c|c|c|c|c|}
\hline $\begin{array}{l}\text { Tumor Characteristics, N } \\
(\%)\end{array}$ & All & NHPI & Asian & White & Other/Unknown \\
\hline \multicolumn{6}{|l|}{ Stage } \\
\hline In situ & $61(15.7)$ & $15(12.7)$ & $25(18.9)$ & $18(14)$ & $3(30)$ \\
\hline Localized & $241(62)$ & $70(59.3)$ & $80(60.6)$ & $87(67.4)$ & $4(40)$ \\
\hline Regional & $87(22.4)$ & $33(28)$ & $27(20.5)$ & $24(18.6)$ & $3(30)$ \\
\hline $\begin{array}{l}\text { Tumor size, mm, mean } \\
\text { (SD) }\end{array}$ & $\begin{array}{l}18.7 \\
(14.5)\end{array}$ & $\begin{array}{l}19.1 \\
(16.8)\end{array}$ & $\begin{array}{l}18.9 \\
(15.4)\end{array}$ & $\begin{array}{l}17.7 \\
(11.3)\end{array}$ & $24.7(11.9)$ \\
\hline \multicolumn{6}{|l|}{ Grade } \\
\hline Well differentiated & $78(20.1)$ & $25(21.2)$ & $25(18.9)$ & $28(21.7)$ & 0 \\
\hline Moderately differentiated & $183(47)$ & $58(49.2)$ & $67(50.8)$ & $55(42.6)$ & $3(30)$ \\
\hline Poorly differentiated & $90(23.1)$ & $25(21.2)$ & $28(21.2)$ & $34(26.4)$ & $3(30)$ \\
\hline Undifferentiated & $12(3.1)$ & $5(4.2)$ & $3(2.3)$ & $4(3.1)$ & 0 \\
\hline Unknown & $26(6.7)$ & $5(4.2)$ & $9(6.8)$ & $8(6.2)$ & $4(40)$ \\
\hline \multicolumn{6}{|l|}{ Lymph node } \\
\hline Positive & $77(19.8)$ & $31(26.3)$ & $22(16.7)$ & $21(16.3)$ & $3(30)$ \\
\hline Negative & $\begin{array}{l}309 \\
(79.4)\end{array}$ & 87 (73.7) & $107(81.1)$ & $108(83.7)$ & $7(70)$ \\
\hline Unknown & $3(0.8)$ & 0 & $3(2.3)$ & 0 & 0 \\
\hline \multicolumn{6}{|l|}{ Estrogen receptor status } \\
\hline Positive & $327(84)$ & $102(86.4)$ & $108(81.8)$ & $109(84.5)$ & $8(80)$ \\
\hline Negative & $52(13.4)$ & $13(11)$ & $19(14.4)$ & $18(14)$ & $2(20)$ \\
\hline Borderline & $1(0.3)$ & $1(0.9)$ & $0(0)$ & $0(0)$ & $0(0)$ \\
\hline Unknown & $9(2.3)$ & $2(1.7)$ & $5(3.8)$ & $2(1.6)$ & $0(0)$ \\
\hline \multicolumn{6}{|l|}{$\begin{array}{l}\text { Progesterone receptor } \\
\text { status }\end{array}$} \\
\hline Positive & $288(74)$ & $93(78.8)$ & $94(71.1)$ & $94(72.9)$ & $7(70)$ \\
\hline Negative & 89 (22.9) & $23(19.5)$ & $31(23.5)$ & $32(24.8)$ & $3(30)$ \\
\hline Borderline & $2(0.5)$ & $0(0)$ & $1(0.8)$ & $1(0.8)$ & $0(0)$ \\
\hline Unknown & $10(2.6)$ & $2(1.7)$ & $6(4.6)$ & $2(1.6)$ & $0(0)$ \\
\hline
\end{tabular}




\begin{tabular}{|llllll|}
\hline $\begin{array}{l}\text { Tumor Characteristics, } \mathbf{N} \\
(\%)\end{array}$ & All & NHPI & Asian & White & Other/Unknown \\
\hline Her2 status & & & & & \\
\hline Positive & $20(5.1)$ & $3(2.5)$ & $10(7.6)$ & $6(4.7)$ & $1(10)$ \\
\hline Negative & $210(54)$ & $66(55.9)$ & $62(47)$ & $77(59.7)$ & $5(50)$ \\
\hline Borderline & $2(0.5)$ & 0 & 0 & $2(1.6)$ & $0(0)$ \\
\hline Unknown & 157 & $49(41.5)$ & $60(45.5)$ & $44(34.1)$ & $4(40)$ \\
\hline
\end{tabular}

Table 3 shows the patients' treatment regimen following surgery. The majority of patients had either surgery followed by radiation or surgery followed by endocrine therapy, $35 \%$ and $31 \%$, respectively. The primary treatment differed by race/ethnic group, with the most common being surgery-endocrine for NHPIs (36\%) and surgery-radiation for Asians (37.9\%) and Whites (38.0\%). Surgery only and surgery followed by chemotherapy were less common primary treatments for the whole cohort, at $18 \%$ and $15 \%$ respectively.

Table 3

Treatment Regimen

\begin{tabular}{|llllll|}
\hline $\begin{array}{l}\text { Treatment order, } \\
\text { N (\%) }\end{array}$ & All & NHPI & Asian & White & Other/Unknown \\
\hline Surgery Only (S) & $71(18.3)$ & $19(16.1)$ & $21(15.9)$ & $27(20.9)$ & $4(40)$ \\
\hline Surgery-Chemotherapy (SC) & $59(15.2)$ & $19(16.1)$ & $18(13.6)$ & $20(15.5)$ & $2(20)$ \\
\hline Surgery-Radiation (SR) & $137(35.2)$ & $38(32.2)$ & $50(37.9)$ & $49(38)$ & 0 \\
\hline Surgery-Endocrine therapy (SE) & $122(31.4)$ & $42(35.6)$ & $43(32.6)$ & $22(25.6)$ & $4(40)$ \\
\hline
\end{tabular}

Weight changes among all 389 patients across six time points, after adjustment for race/ethnicity, age and BMI at surgery are displayed in Fig. 2. The mean predicted probability for patients maintaining their weight $<3$ months from surgery was over $90 \%$; however, this percent steadily decreased to $44 \%$ at $36-47$ months, with weight loss and weight gain at $40 \%$ and $16 \%$, respectively. We compared this to the normal MEC cohort population with similar demographics as the MEC-KPHI breast cancer patients according to ethnicity (Hawaiian, Japanese = Asian, White), age range, BMI range, and first year of diagnosis (2003) to match the year of MEC data collection. We saw significant differences in weight change between the two groups. Based on polytomous logistic model with outcomes loss/stable/gained weight, fixed effects for time (5 groups, $p<0.0001)$, age $(<65,65-74,75+, p<0.0001)$, race (Hawaiian, Japanese, White, $p<$ $0.0001), \mathrm{BMI}(<25,25-29.9,30-34.9,35+, \mathrm{p}<0.0001)$, during the time period, $34 \%$ of MEC participants lost weight compared to $40 \%$ of MEC-KPHI patients during the same time period. Figure 2 . Weight Changes from Surgery to Up to 4 Years 
Additionally, weight changes across time by age at surgery (groups divided at the median age 71 years), race/ethnicity, and BMI groups are shown In Figs. 2A, 2B, 2C, respectively.

Age at surgery ( $\leq 71$ vs $>71$ years, see Fig. $2 A$ ): The mean predicted probability of patients who maintained their weight steadily decreased across time for both age groups, though lower percentages were observed in patients age $\leq 71$ years at surgery compared to patients age $>71$ years. A higher percentage of patients who were age $\leq 71$ years at surgery gained weight across time.

Race/Ethnicity (NHPI, Asians, Whites, Others, see Fig. 2B): The percentage of patients who had stable weight decreased across time across all race/ethnic groups. NHPIs and Whites, and to a lesser extent Asians, showed similar profiles, however NHPI had a higher percentage of patients who gained weight across time compared to Whites and Asians.

BMl at surgery $\left(<25,25-29.9,30-34.9,35+\mathrm{kg} / \mathrm{m}^{2}\right.$, see Fig. $\left.2 \mathrm{C}\right)$ : Across all BMl groups, the percentage of patients who maintained their weight decreased across time. More patients with BMI $30+\mathrm{kg} / \mathrm{m}^{2}$ (at surgery) loss weight across time compared to patients with $\mathrm{BMI}<30 \mathrm{~kg} / \mathrm{m}^{2}$.

(S) Surgery alone (see Fig. 3A): The percentage of patients who maintained a stable weight from surgery generally decreased over time ( $89 \%$ at $<3$ months to $52 \%$ at $36-47$ months); patients with stable weight comprise the majority at each time point. Weight loss continued throughout the evaluated time frame and peaked $24-35$ months after surgery at $41 \%$. The percentage of patients with weight gain was generally consistent throughout this timeframe. We also ran the model according to surgery type (lumpectomy, mastectomy, other), with age, race, and BMl, and found that surgery type was not significant $(p=0.89)$, and the weight change profiles were similar.

(SC) Surgery-Chemotherapy (see Fig. 3B): From 3 months on, the percentage of patients who maintained weight after surgery was generally constant at roughly $50 \%$. Within 3 months after surgery, a time when patients routinely receive chemotherapy, $16 \%$ of patients had significant weight loss, and over $30 \%$ of patients had weight loss at each subsequent time point. Weight gain continued to steadily increase during this timeframe and peaked $12-23$ months after surgery at $14 \%$. Weight gain was less commonly seen than weight loss throughout the post-surgery period.

(SR) Surgery-Radiation (see Fig. 3C): The percentage of patients who maintained a stable weight from surgery consistently decreased over time ( $94 \%$ at $<3$ months to $38 \%$ at $36-47$ months), and the percentage of patients who loss or gained weight steadily increased after initial surgery. Weight loss was about 2-fold more common than weight gain at each time point.

(SE) Surgery-Endocrine (see Fig. 3D): The pattern of weight change in this patient group was very similar to the pattern in the surgery-radiation patient group, where the percentage of patients with stable weight decreased over time. 
Weight changes from surgery were similar between the treatment groups of $S$, SR and SE across time ( $p$ $=0.89$ based on a $F$ test with 20 degrees of freedom). A similar model that included all 389 patients and a modified treatment term, collapsing S, SR, SE into one group (vs SC), resulted in a significant interaction term between time and treatment ( $p=0.0008$ based on a $F$ test with 10 degrees of freedom). Patients who underwent SC treatment experienced different weight changes compared to S, SR, and SE groups; they had the highest percent weight loss, particularly within the first 3 months after surgery, while they received chemotherapy. This percentage with weight loss plateaued at 3-5 months and continued for the remainder of the timeframe, up to 4 years after surgery.

\section{Discussion}

We evaluated 389 early stage MEC/KPHI breast cancer patients' weight changes for 4 years after surgery according to treatment-chemotherapy, radiation, endocrine therapy or surgery alone-adjusted for age, race/ethnicity and initial BMI. Our overall population is older than the median age of breast cancer diagnosis, due to the MEC age criteria of 45-75 in the mid-90's and is racially/ethnically diverse. Consistent with known racial/ethnic disparities, this group of NHPI patients had proportionately more regional stage and lymph node positive disease, compared to Asian and White patients. When adjusted for age at diagnosis, race/ethnicity and initial BMI, we saw treatment related weight change differences. Overall, all treatment groups (S, SC, SR, SE) showed that a majority of patients had stable weight. However over time, weight loss compared to weight gain continued to increase and was comparatively over 2-fold higher.

We suspect the ongoing weight loss in all treatment groups to be at least in part due to sarcopenia. This can naturally occur with aging and is further exacerbated in older adults with cancer. There are recent efforts to focus on body composition evaluation using CT technology and correlate metrics of skeletal muscle mass, visceral adipose tissue, subcutaneous adipose tissue and others with breast cancer outcomes $(20,21)$. For breast cancer patients receiving chemotherapy, sarcopenia has been found a predictor of adverse events (22). Also, in a recent meta-analysis, sarcopenia was associated with not only severe chemotherapy toxicity as well as shorter overall survival and time to tumor progression. All together this suggests that in clinical practice, body composition assessment could be valuable as a prognostic parameter in breast cancer. Despite being a risk factor for mortality among early stage female breast cancer patients, there are no guidelines for ongoing or initial evaluation of patients for sarcopenia. Clinical trial interventions evaluating the impact of diet and physical activity on weight gain after a breast cancer diagnosis have been underway (23-25). More recent interventions are utilizing body composition analysis to evaluate improvement in muscle mass and function in breast cancer survivors. We have a tailored exercise program for breast cancer patients, irrespective of their current treatment, and have utilized body composition metrics to evaluate changes and correlate them with disease outcomes (NCT04013568).

The majority of prior studies evaluated weight change in early stage breast cancer at least 6 months post diagnosis. In our study we utilized all available weights and assigned them to established treatment time 
points. Despite completing a majority of adjuvant treatments (chemotherapy and radiation) by 12 months after surgery, continued weight loss was observed in all treatment groups. The differences between weight loss and weight gain increased and worsened from 12 months to 48 months for the treatments of surgery (S), surgery-radiation (SR) and surgery-endocrine (SE).

The surgery-chemotherapy (SC) treatment group showed significant differences between weight loss and weight gain initially, beginning at month 3 that persisted to 48 months, indicating a significant difference from other treatment groups.

This initial steep increase in percent of patients with weight loss for those who received SC, reveals a potentially important therapeutic window for intervention. In practice, patients who are undergoing chemotherapy are encouraged to stay physically active as tolerated during their treatment; however there are no established guidelines that are commonly recommended. Often due to chemotherapy toxicities including fatigue, nausea/vomiting, and decreased appetite, patients struggle to be physically active and this can further contribute to deconditioning. Our group is assessing a tailored exercise program for breast cancer patients undergoing various treatments (S, SR, SE, SC). Other studies have evaluated physical activity regimens during chemotherapy and found improvements in cardiorespiratory fitness (26) chemotherapy related fatigue (27) and less nausea and pain (28). Our data suggest the initial 3 months post initial surgical intervention to be the most dynamic and potentially important period for addressing weight loss changes in patients with early stage breast cancer undergoing chemotherapy. Efforts should be made to utilize local physical activity programs and/or accrue to available clinical trials that intervene during this timeframe.

Strengths and Limitations:

Our study utilizes a substantial amount of data from three independent and unique sources: HI MEC, KPHI and HTR. Several coding sources (ICD-9/10, CPT, HCPC, local Kaiser codes) in the KPHI EMR were used to identify breast cancer treatment (surgery, chemotherapy, radiation, and endocrine therapy). Through a comprehensive review of all procedure and prescription codes in patients' EMR, 6 months prior to their first encounter/diagnosis date and up to 1 year after, as well as manual chart review for some, we appropriately classified the different types of breast cancer treatment, despite each type being coded in a variety of ways. With data available at each patient encounter, we also had access to an abundance of data, including enrollment, demographics, diagnosis, and detailed treatment. Diagnosis data from KPHI EMR and HTR were generally consistent as well. In addition, our diverse and older population fills a gap currently lacking in the literature.

There are some limitations however, our study did not obtain information regarding whether there was intentionality for weight loss. Because these data were adjusted for initial BMI and still weight loss was more prevalent than weight gain, we conclude that this trend represents all patients-overweight, underweight and normal weight at initial diagnosis. Although we have some information on physical activity and dietary habits from the MEC participants at date of cohort entry and 10 years afterward, we do not have information on specific behavioral changes occurring during treatment. Additionally our 
observation period post treatment is up to 4 years, which may not capture a long enough time frame to conclude effects on breast cancer survivors. Also, no recurrence data was available to evaluate if weight changes had any effect on disease free survival in this cohort.

\section{Conclusions}

Our study found treatment related differences in weight changes irrespective of age, race/ethnicity and initial BMI. Although the majority of patients had stable weight after initial surgery, weight loss increased irrespective of treatment, S, SC, SR, SE. Given the adverse consequences of weight gain and weight loss after breast cancer diagnosis, particularly for those undergoing chemotherapy, identifying interventional strategies during treatment may positively influence outcomes. Targeted weight management interventions should be utilized during and after treatment to promote overall health and long term survivorship.

\section{Abbreviations}

EMR-Electronic Medical Record

HI-MEC- Hawaii Multiethnic Cohort

HTR-Hawaii Tumor Registry

KPHI- Kaiser Permanente Hawaii

NHPI-Native Hawaiian/Pacific Islanders

\section{Declarations}

Ethics approval and consent to participate

The Institutional Review Boards at the University of Hawaii and Kaiser Permanente Hawaii approved this study. All aspects of the investigation complied with

current rules for research involving Human Subjects.

Consent for publication

Not applicable

Availability of data and material

The datasets used and/or analyzed during the current study are available from the corresponding author on reasonable request. 
Competing interests

The authors declare that they have no completing interests.

\section{Funding}

This work was completed as part of the funding from the University of Hawaii Cancer

Center P30 CA071789 Grant from the National Institutes of Health (J.Fukui, K.White,

L. Wilkens). The funding body had no role in the design; in the collection, analysis, or interpretation of data; in writing of the manuscript; or in the decision to submit the manuscript for publication.

\section{Authors' contributions}

All authors made substantial contribution to conception and design, analysis and interpretation of data, and critical review of the manuscript. JF conceived of the study, coordinated the data acquisition and analysis, generated table and figures and helped to draft the manuscript. KW and LW contributed to the statistical methods, data analysis, generated table and figures and helped to draft the manuscript. TF and CO contributed to the data analysis and helped to draft the manuscript. All authors read and approved the final manuscript.

\section{Acknowledgements}

The authors would like thank the participants in the Hawaii Multiethnic Cohort (HI-MEC)

for their willingness to be included in an important cohort that supports research such as this study.

\section{References}

1. Playdon MC, Bracken MB, Sanft TB, et al. Weight gain after breast cancer diagnosis and all-cause mortality: systematic review and meta-analysis. JNCl J Natl Cancer Inst. 2015. doi:10.1093/jnci/djv275.

2. Protani M, Coory M, Martin JH. Effect of obesity on survival of women with breast cancer: systematic review and meta-analysis. Breast Cancer Res Treat. 2010. doi:10.1007/s10549-010-0990-0.

3. Sparano JA, Wang M, Zhao F, et al. Obesity at diagnosis is associated with inferior outcomes in hormone receptor-positive operable breast cancer. Cancer. 2012. doi:10.1002/cncr.27527.

4. Nyrop KA, Deal AM, Shachar SS, et al. Weight trajectories in women receiving systemic adjuvant therapy for breast cancer. Breast Cancer Res Treat. 2020. doi:10.1007/s10549-019-05501-8.

5. Raghavendra A, Sinha AK, Valle-Goffin J, et al. Determinants of weight gain during adjuvant endocrine therapy and association of such weight gain with recurrence in long-term breast cancer 
survivors. Clin Breast Cancer. 2018. doi.org/10.1016/j.clbc.2017.11.006.

6. Vagenas D, DiSipio T, Battistutta D, et al. Weight and weight change following breast cancer: evidence from a prospective, population-based, breast cancer cohort study. BMC Cancer. 2015. doi:10.1186/s12885-015-1026-2.

7. Nyrop KA, Deal AM, Lee JT, et al. Weight changes in postmenopausal breast cancer survivors over 2 years of endocrine therapy: a retrospective chart review. Breast Cancer Res Treat. 2017. doi:10.1007/s10549-017-4106-y.

8. Schvartsman G, Gutierrez-Barrera AM, Song J, et al. Association between weight gain during adjuvant chemotherapy for early-stage breast cancer and survival outcomes. Cancer Med. 2017. doi:10.1002/cam4.1207.

9. Dunne RF, Loh KP, Williams GR, et al. Cachexia and sarcopenia in older adults with cancer: a comprehensive review. Cancers (Basel). 2019. doi:10.3390/cancers11121861.

10. Williams GR, Chen Y, Kenzik KM, et al. Assessment of sarcopenia measures, survival, and disability in older adults before and after diagnosis with cancer. JAMA Netw Open. 2020. doi:10.1001/jamanetworkopen.2020.4783.

11. Xia L, Zhao R, Wan Q, et al. Sarcopenia and adverse health-related outcomes: An umbrella review of meta-analyses of observational studies. Cancer Med. 2020. doi:10.1102/cam4.3428.

12. Zhang $X M$, Dou QL, Zeng $Y$, et al. Sarcopenia as a predictor of mortality in women with breast cancer: a meta-analysis and systematic review. BMC Cancer. 2020. doi:10.1186/s12885-020-6645-6.

13. Del Fabbro E, Parsons H, Warneke CL, et al. The relationship between body composition and response to neoadjuvant chemotherapy in women with operable breast cancer. Oncologist. 2014. doi:10.1634/theoncologist.2012-0169.

14. Cespedes EM, Kroenke $\mathrm{CH}$, Bradshaw PT, et al. Post-diagnosis weight change and survival following a diagnosis of early stage breast cancer. Cancer Epidemiol Biomarkers Prev. 2017. doi:10.1158/1055-9965.EPI-16-0150.

15. Chen X, Lu W, Gu K, et al. Weight change and its correlates among breast cancer survivors. Nutr Cancer. 2011. doi:10.1080/01635581.2011.539316.

16. Hawaii Tumor Registry 2012-2016. Accessed 20 Jan 2021. https://www.uhcancercenter.org/research/shared-resources/hawaii-tumor-registry.

17. Kolonel LN, Henderson BE, Hankin JH, et al. A multiethnic cohort in Hawaii and Los Angeles: baseline characteristics. Am J Epidemiol. 2000. doi:10.1093/oxfordjournals.aje.a010213.

18. Yost K, Perkins $\mathrm{C}$, Cohen $\mathrm{R}$, et al. Socioeconomic status and breast cancer incidence in California for different race/ethnic groups. Cancer causes control. 2001. doi:10.1023/a:1011240019516.

19. Yang J, Schupp CS, Harrati A, et al. Developing an area-based socioeconomic measures from American Community Survey data. California: Cancer Prevention Institute of California, Fremont; 2014. 
20. Rossi F, Valdora F, Bignotti B, et al. Evaluation of body Computed Tomography-determined sarcopenia in breast cancer patients and clinical outcomes: A systematic review. Cancer Treat Res Commun. 2019. doi:10.1016/j.ctarc.2019.100154.

21. Deluche E, Leobon S, Desport JC, et al. Impact of body composition on outcome in patients with early breast cancer. Support Care Cancer. 2018. doi:10.1007/s00520-017-3902-6.

22. Aleixo GFP, Williams GR, Nyrop KA, et al. Muscle composition and outcomes in patients with breast cancer: meta-analysis and systematic review. Breast Cancer Res Treat. 2019. doi:10.1007/s10549019-05352-3.

23. Chlebowski RT, Blackburn GL, Thompson CA, et al. Dietary fat reduction and breast cancer outcome: interim efficacy results from the Women's Intervention Nutrition Study. J Natl Cancer Inst. 2006. doi:10.1093/jnci/djj494.

24. Holmes $M$, Chen W, Feskanich $D$, et al. Physical activity and survival after breast cancer diagnosis. JAMA. 2005. doi:10.1001/jama.293.20.2479.

25. Dieli-Conwright CM, Courneya KS, Demark-Wahnefried W, et al. Effects of aerobic and resistance exercise on metabolic syndrome, sarcopenia obesity, and circulating biomkarker in overweight or obese survivors of breast cancer: a randomized controlled trial. J Clin Oncol. 2018. doi:10.1200/JCO.2017.75.7526.

26. Vincent $F$, Deluche $E$, Boniset $J$, et al. Home-based physical activity in patients with breast cancer during and/or after chemotherapy impact on cardiorespiratory fitness. A 3-Arm randomized controlled trial (APAC). Integr Cancer Ther. 2020. doi:10.1177/1534735420969818.

27. Wengström Y, Bolam KA, Mijwel S, et al Optitrain: a randomised controlled exercise trial for women with breast cancer undergoing chemotherapy. BMC Cancer. 2017.

28. doi:10.1186/s12885-017-3079-x.

29. van Wart $H$, Stuiver MM, van Harten $W H$, et al. Effect of low-intensity physical activity and moderateto high-intensity physical exercise during adjuvant chemotherapy on physical fitness, fatigue, and chemotherapy completion rates: results of the PACES randomized clinical trial. J Clin Oncol. 2015. doi:10.1200/JC0.2014.59.1081.

\section{Figures}




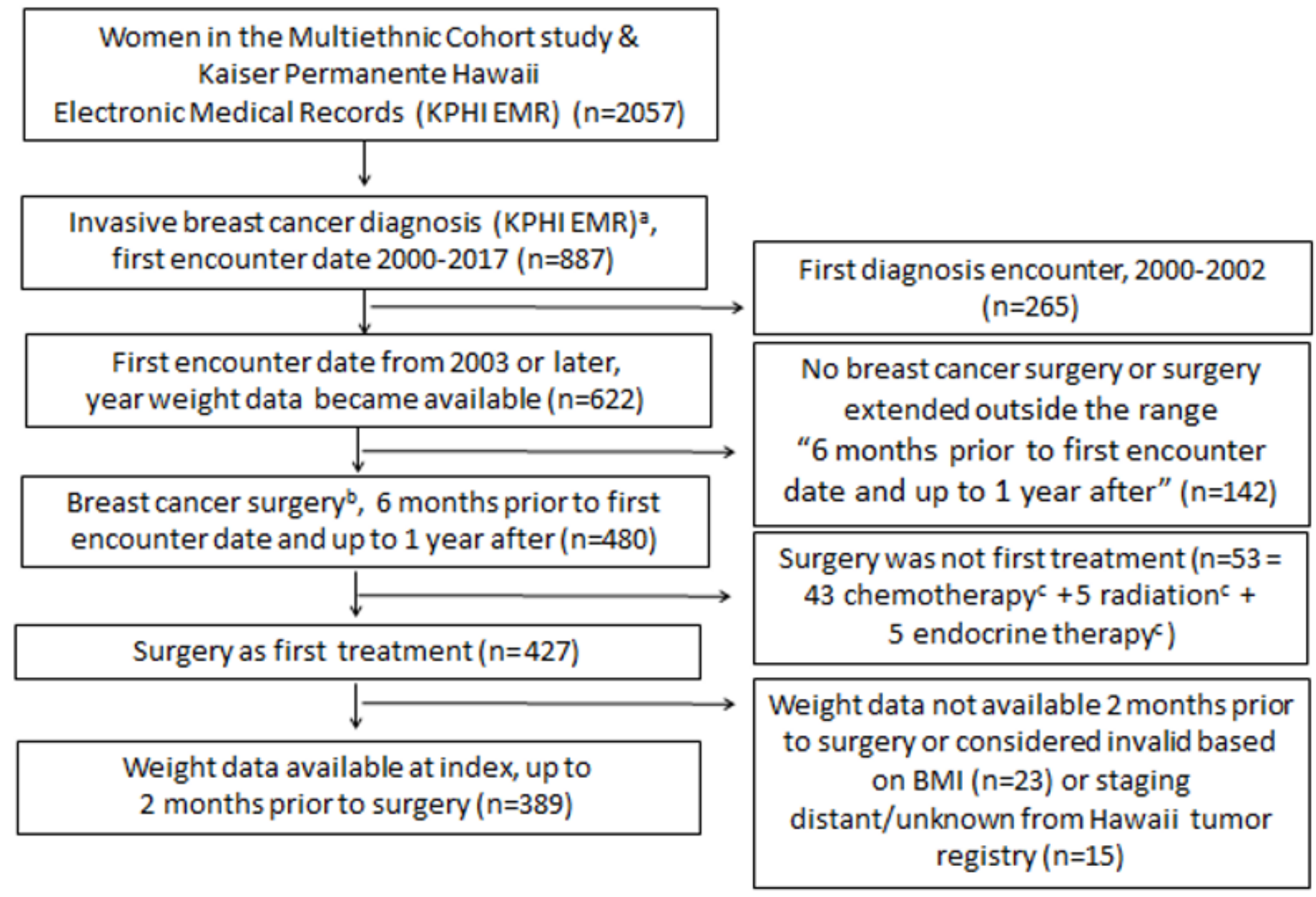

Figure 1

Flowchart of Hawaii cases from the Multiethnic Cohort (MEC)-Kaiser linked dataset Study sample selection. aDefined using International Classification of Diseases (ICD) 9th/10th revision codes C50, 174, Z85.3, V10.3. bDefined using ICD-9/10, Current Procedural Terminology (CPT), and local Kaiser Permanente Hawaii (KPHI) codes. c Defined using ICD 9/10, CPT, Healthcare Common Procedure Codes, Revenue codes, and National Drug Codes. 

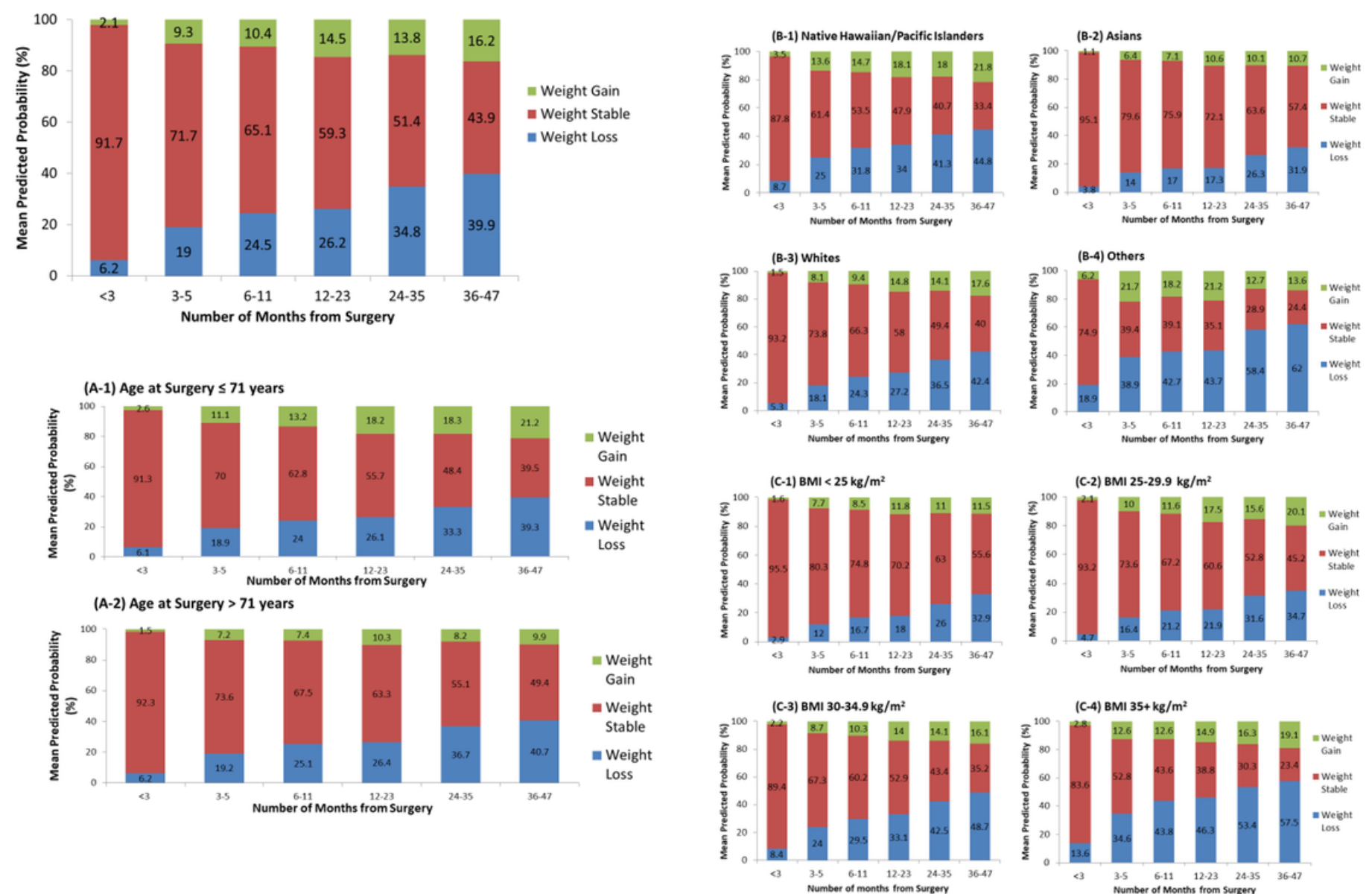

\section{Figure 2}

Weight changes from surgery based on generalized odds logistic model with outcomes loss/stable/gained weight, random intercept term for each patient, and fixed effects for time (6 groups, $p<0.0001)$, age at surgery $(<65,65-74,75+, p=0.0009)$, race (Hawaiian, Asian, White, Other/unknown, $p=0.041), B M l$ at surgery $(<25,25-29.9,30-34.9,35+, p=0.0009)$. Weight changes from surgery based on generalized odds logistic model described in Figure 2. Number of months from surgery ( $<3$ months, 3-5, 611 , 12-23, 24-35, 36-47 months): Fig 2A-1, Age $\leq 71$ years; Fig 2A-2, Age > 71 years Weight changes from surgery based on generalized odds logistic model described in Figure 2. Number of months from surgery (<3 months, 3-5, 6-11 , 12-23, 24-35, 36-47 months): Fig 2B-1, Hawaiians; Fig 2B-2 Asians; Fig 2B-3, Whites; Fig 2B-4, Others Weight changes from surgery based on generalized odds logistic model described in Figure 2. Number of months from surgery (<3 months, 3-5, 6-11 , 12-23, 24-35, 36-47 months): Fig 2C-1, BMl< 25 kg/m2; Fig 2C-2, BMI 25-29.9 kg/m2 ; Fig 2C-3, BMI 30-34.9 kg/m2 ; Fig 2C-4, BMl $35+\mathrm{kg} / \mathrm{m} 2$ 
(A) Surgery alone

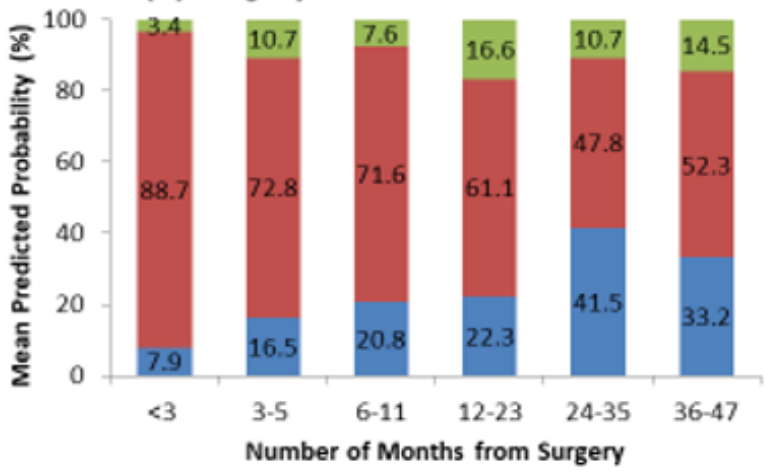

(C) Surgery-Radiation

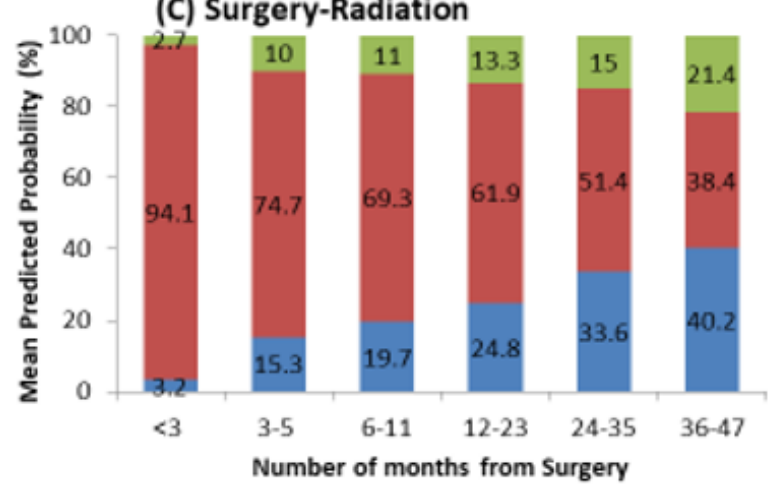

(B) Surgery-Chemotherapy

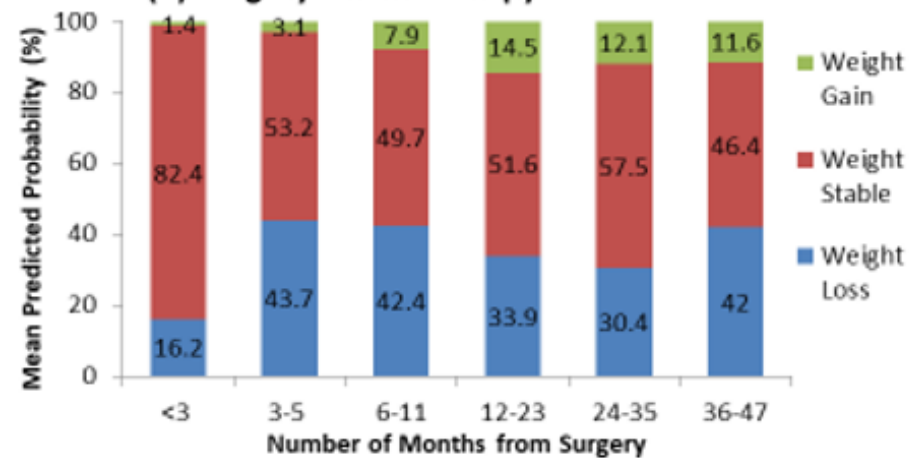

(D) Surgery-Endocrine Therapy

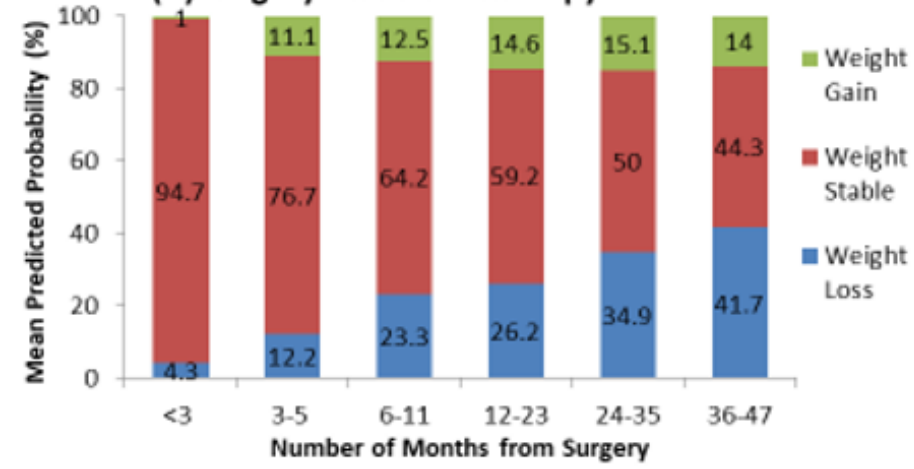

\section{Figure 3}

Weight changes from surgery based on logistic model with outcomes loss/stable/gained weight, random intercept term for each patient, fixed effects for time (6 groups, $p<0.0001)$, age at surgery $(<65,65-74,75+$, $p=0.0004)$, race (Hawaiian, Asian, White, Other/unknown, $p=0.055), B M l$ at surgery $(<25,25-29.9,30-34.9$, $35+, p=0.0009)$, treatment (Surgery alone (S), Surgery-Chemotherapy (SC), Surgery-Radiation (SR),

Surgery-Endocrine therapy (SE), $p=0.073)$, interaction of time and treatment $(p=0.074)$.; Fig 3B-SC; Fig 3CSR; Fig 3D-SE 